\title{
THERMAL TRANSPORT BEHAVIOR OF A LIQUID PLUG MOVING INSIDE A DRY CAPILLARY TUBE
}

\author{
Ashish Kumar Bajpai E Sameer Khandekar* \\ Department of Mechanical Engineering, Indian Institute of Technology \\ Kanpur, Kanpur 208016, India
}

*Address all correspondence to: Sameer Khandekar, E-mail: samkhan@iitk.ac.in.

\begin{abstract}
Computational simulation of flow and heat -transfer characteristics of a single isolated liquid plug (of glycerin and water, respectively) flowing in a round capillary tube (internal diameter, $D=2.0 \mathrm{~mm}$ ), so as to understand its local thermo-hydrodynamic transport behavior, is reported. Both, constant heat flux and constant-temperature boundary conditions have been considered at the tube wall. When an isolated liquid plug moves in a capillary tube, there is a difference in the advancing and receding dynamic contact angles of the two menisci, respectively. This has been considered in the simulations. The linearized simplification of Hoffman-Tanner's law is used to model the variation in the two respective apparent dynamic contact angles with the velocity of the liquid plug (i.e., $\mathrm{Ca}=\mu U / \sigma$ ). Simulations are carried out for a range of Capillary numbers and lengths of liquid plugs ( $L / D$ ratio). It has been found that variation in $d y$ namic contact angle leads to an enhanced local and average heat-transfer coefficient in the moving liquid plug; the local fluid circulation being affected by menisci deformation. In addition, as the length of the liquid plug is increased, the heat-transfer coefficient decreases and finally shows the asymptotic transport behavior of Poiseuille flow. Other than the flow Capillary number, the fluid Prandtl number and the $L / D$ ratio of the liquid plug, strongly affect the local thermo hydrodynamics.
\end{abstract}

KEY WORDS: liquid plug flow, Taylor bubble, contact angle hysteresis, capillary tube, transport coefficients, pulsating heat pipe

\section{INTRODUCTION}

Microscale two-phase flow is an active research area because of its application in many engineering devices such as electronics cooling, microreactors, catalyst coating in capillaries, refrigeration industry, porous media systems, etc. (Gunther et al., 2004; Kolb and Cerro, 1991; Zheng et al., 2007). Taylor flow or capillary slug flow, characterized by long bubble slugs separated by liquid plugs, constitutes the typical regime under such conditions (Fairbrother and Stubbs, 1935; 


\section{NOMENCLATURE}

\begin{tabular}{|c|c|c|c|}
\hline $\begin{array}{l}\mathrm{Bo} \\
\mathrm{Ca}\end{array}$ & $\begin{array}{l}\text { Bond number }\left[D^{2} g\left(\rho_{1}-\rho_{\mathrm{v}}\right) / \sigma\right] \\
\text { capillary number }[\mu U / \sigma]\end{array}$ & $x^{*}$ & $\begin{array}{l}\text { nondimensional position in } X \\
\text { direction }\end{array}$ \\
\hline $\mathrm{C}_{\mathrm{p}}$ & specific heat (J/kg-K) & $y^{*}$ & nondimensional position in $Y$ \\
\hline$D$ & liquid plug diameter $(\mathrm{m})$ & & direction \\
\hline$g$ & $\begin{array}{l}\text { acceleration due to gravity } \\
\left(\mathrm{m} / \mathrm{s}^{2}\right)\end{array}$ & $z^{*}$ & $\begin{array}{l}\text { nondimensional position in } Z \\
\text { direction }\end{array}$ \\
\hline$h$ & $\begin{array}{l}\text { heat-transfer coefficient (W/ } \\
\left.\mathrm{m}^{2}-\mathrm{K}\right)\end{array}$ & \multicolumn{2}{|c|}{ Greek Symbols } \\
\hline$J$ & total volumetric flux $(\mathrm{m} / \mathrm{s})$ & $\mu$ & dynamic viscosity (Pa-s) \\
\hline$k$ & thermal conductivity $(\mathrm{W} / \mathrm{mK})$ & $\rho$ & density $\left(\mathrm{kg} / \mathrm{m}^{3}\right)$ \\
\hline$L$ & liquid plug length (m) & $\sigma$ & surface tension $(\mathrm{N} / \mathrm{m})$ \\
\hline $\begin{array}{l}\mathrm{Nu} \\
P\end{array}$ & Nusselt number $\left(h D / k_{1}\right)$ & $\theta$ & contact angle (rad) \\
\hline $\begin{array}{l}P \\
\operatorname{Pr}\end{array}$ & $\begin{array}{l}\text { pressure }\left(\mathrm{N} / \mathrm{m}^{2}\right) \\
\text { Prandtl number }\left(\mu C_{\mathrm{p}} / k_{1}\right)\end{array}$ & $\Psi$ & dimensionless bubble \\
\hline$q^{\prime \prime}$ & heat flux (W) & & velocity (-) \\
\hline$T$ & $\begin{array}{l}\text { temperature }\left({ }^{\circ} \mathrm{C} \text { or } \mathrm{K}\right) \\
\text { time }(\mathrm{s})\end{array}$ & \multicolumn{2}{|c|}{ Subscripts } \\
\hline$t^{*}$ & $\begin{array}{l}\text { nondimensional time }[t U / \\
(D+2 L)]\end{array}$ & $\begin{array}{l}a \\
d\end{array}$ & $\begin{array}{l}\text { advancing } \\
\text { dynamic }\end{array}$ \\
\hline$t s$ & time step & $\mathrm{m}$, avg & mean/average \\
\hline$U$ & velocity of liquid plug $(\mathrm{m} / \mathrm{s})$ & $\mathrm{r}$ & receding \\
\hline$U_{\mathrm{b}}$ & velocity of bubble $(\mathrm{m} / \mathrm{s})$ & $\mathrm{s}$ & static \\
\hline$u^{*}$ & nondimensional velocity in $X$ & $\mathrm{~W}$ & wall \\
\hline & direction & 1 & liquid \\
\hline$\vec{V}$ & velocity vector $(\mathrm{m} / \mathrm{s})$ & $\mathrm{p}$ & plug \\
\hline$v^{*}$ & $\begin{array}{l}\text { nondimensional velocity in } Y \\
\text { direction }\end{array}$ & $\begin{array}{l}\mathrm{v} \\
\mathrm{b}\end{array}$ & $\begin{array}{l}\text { vapor } \\
\text { bubble }\end{array}$ \\
\hline$w$ & $\begin{array}{l}\text { nondimensional velocity in } Z \\
\text { direction }\end{array}$ & & \\
\hline
\end{tabular}

Taylor, 1961; Bretherton, 1961; Cox, 1964; Thulasidas et al., 1997). Many researchers have worked with the Taylor flow regime and tried to understand the thermo-hydrodynamic properties of such flows (Oliver and Wright, 1964; Chen, 1986; Schwartz et al., 1986; Fermigier and Jennfer, 1991). A review of local hydrodynamics of such flows, especially in the context of pulsating heat pipes, can be found in Khandekar et al. (2010). Internal circulations in liquid plugs have been found to be the major cause of enhanced heat (and/or mass) transfer in Tay- 
lor flow (Thulasidas et al., 1997). However, not many studies have reported the dynamic and local, meniscus-level deformations due to the imposed flow velocity and its subsequent impact on sensible heat-transport behavior of Taylor flows.

Fairbrother and Stubbs (1935) were one of the first to study such kinds of flows. They performed experiments to determine liquid flow rates through a capillary by movement of an indicator bubble. They confirmed the existence of a liquid thin film between the bubble and the tube wall which was the prime reason for higher velocity of the bubble compared to that of the liquid. Taylor (1961) refined Fairbrother's experiments for a wide range of capillary numbers $(\mathrm{Ca}=$ $\mu U / \sigma)$ and reported values of relative bubble velocity. Since then, many researchers have tried to explore this flow extensively and yet, because of the complexity of the problem, its thermo-hydrodynamic characteristics have not been fully discerned until now.

Because of its unique flow behavior, two-phase Taylor flow has proved to be a very promising method of enhancing heat/mass transport compared to singlephase flow. Oliver and Wright (1964) conducted heat-transfer experiments in a 0.25 inch copper tube under a variety of two-phase flow conditions and reported 2.5 times higher heat transfer in two-phase flow compared to that of a singlephase flow regime. Thulasidas et al. (1997) performed experiments in a vertical circular and square capillary tube with air and two different liquids, namely, water and silicon-oil, for a range of capillary numbers. They used high-speed video imaging to characterize liquid flow patterns and PIV (particle imaging velocimetry) for obtaining velocity profiles in liquid plugs. Videos were recorded in a reference frame moving with bubbles and they reported different flow patterns based on the relative liquid and bubble velocity. Depending on the capillary number of the flow, internal circulations were observed which vanish at higher relative velocity. This was the first work which accurately gave the velocity profile in the liquid plug and defined the recirculation time in the vortex.

Later, Triplett et al. (1999) performed experiments in circular and semitriangular microchannels and observed bubbly, slug, slug-annular, churn, and annular flow depending on liquid and gas superficial velocity. Zhao and Bi (2001) investigated the two-phase flow pattern in vertical equilateral triangular section channels and observed a capillary bubbly flow pattern at the smallest dimension of the channel. This pattern, characterized by a train of ellipsoidal-shaped bubbles almost spanning the entire channel cross section, led to the sidewalls being partially dried out at low liquid flow rates.

Apart from extensive experimental research, Taylor flow has also been studied numerically using different techniques like the volume-of-fluid (VOF) meth- 
od, level-set (LS) method, etc. (He et al., 2010; Mehdizadeh et al., 2011). Researchers have tried to capture the liquid film using a high-resolution grid at the cost of increased computation time, but not many studies are available.

One of the characteristics of such flow is the formation of liquid film around the bubble, which is the prime reason for higher bubble velocity. However, at low liquid flow rates, a dry-out situation can also occur where liquid-gas-solid, all the three phases, remain in contact and a three-phase contact line is formed. Serizawa et al. (2002), Cubaud and Ho (2004), and various other researchers (Zhao and Bi, 2001; He et al., 2010) have reported dry-out conditions at low liquid flow rates. He et al. (2010) carried out two-dimensional axis-symmetric numerical simulations of two-phase flow using the phase-field method and they also observed the possibility of a dry interface along with a wet interface with similar conditions, as reported by Serizawa et al. (2002) and Cubaud and Ho (2004).

Hence, at low liquid flow rate, a dry-plug flow regime is observed rather than a wet-plug flow regime. In a dry-plug flow regime, no liquid film exists around the "bubble"; in fact, there is no bubble at all. Figures 1(a) and 1(b), adapted from Lee and Lee (2008), shows typical flow conditions in a wet-plug flow and dry-plug regime, respectively. Figures $1(\mathrm{c})$ and 1(d) show the dry-plug condition in static and dynamic mode. Because of the existence of a three-phase contact line in a dry-plug flow case, a proper modeling of dynamic contact angle is necessary for its analysis, as dynamic contact angle hysteresis is likely to play an important role in the thermo-hydrodynamic behavior of such flows. As can be seen in the figure, when the plug is moving with a constant velocity, the dynamic advancing $\left(\theta_{\mathrm{a}}\right)$ and receding $\left(\theta_{\mathrm{r}}\right)$ contact angles are different than the corresponding static contact angle $\left(\theta_{\mathrm{s}}\right)$ (Berthier, 2008). Fang et al. (2008) numerically studied the evolution of liquid plug flow with sidewall liquid injection by including the contact angle hysteresis in order to correctly model the wall adhesion forces. Their study revealed the importance of contact angle hysteresis on flow pattern and subsequent evolution of flow. Hence, to get an accurate result in the case of dry-plug flow, one needs to carefully model the local dynamic contact angle at the wall.

Fermigier and Jenffer (1991) experimentally studied the effect of contact angle on a two-phase flow pattern in a capillary tube by testing adiabatic air-water mixtures at different flow rates in a tube diameter of $1.6 \mathrm{~mm}$, made of different materials, to include the effect of surface wettability. It was found that a new flow regime, Rivulet flow, replaces the wavy flow at large contact angle. In addition, the transition boundaries for a nonwetting capillary tube system were also significantly different. 
(a)

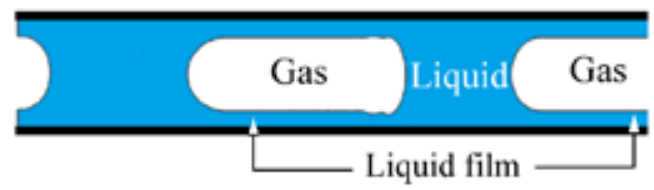

(c)

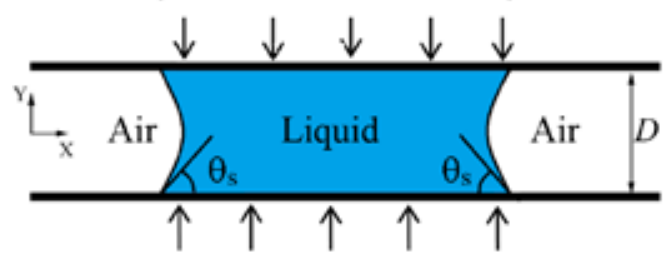

Constant $q^{\prime \prime}$ or $T$ (b)

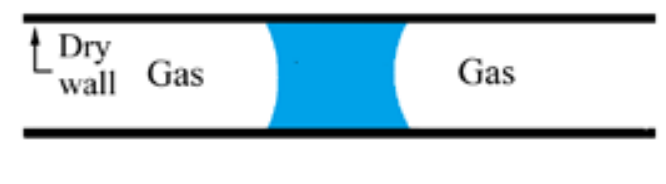

(d)

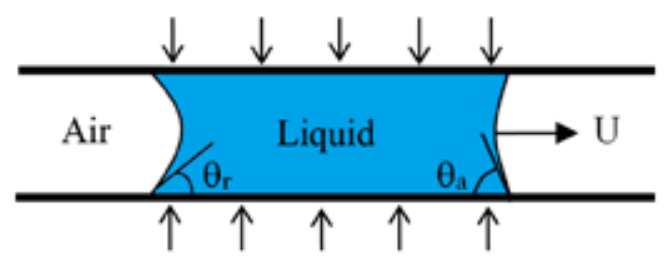

Constant $q^{\prime \prime}$ or $T$

FIG 1: Two configurations of gas-liquid flow in a capillary tube: (a) wet-plug flow regime and (b) dry-plug flow regime. Details of the isolated liquid plug inside a capillary tube. (c) Static liquid plug, with static apparent contact angle $\theta$ s. (d) Plug moving with a constant velocity $\mathrm{U}$, where $\theta \mathrm{a}$ is the advancing and $\theta \mathrm{r}$ the receding contact angle.

Lee and Lee (2008) recently analyzed the effect of surface wettability on pressure drop in round minichannels by conducting experiments with air and water on three different tube materials, i.e., glass, polyurethane, and Teflon, with the inner diameter ranging from 1.62 to $2.16 \mathrm{~mm}$. It was found that in the wet-plug flow regime, moving contact lines did not have much effect, whereas in the dryplug flow regime, the energy dissipation by the moving three-phase contact lines played an important role in determining the pressure drop. A modified LockhartMartinelli-type correlation, to fit the experimental data, was proposed. Rosengarten et al. (2006) studied the effect of contact angle on convective heat transfer in microchannels and found an increase in heat-transfer rate with contact angle.

Though numerous studies are available on the effect of contact angle on two-phase flow, not much work has been carried out to understand the effect of contact angle hysteresis in dry-plug flow regimes, although its importance is reflected by many authors (Barajas and Panton, 1992; Lee and Lee 2008; Fang et al., 2008; Rosengarten et al., 2006; Gupta et al., 2009). The present work is an attempt to address this problem, by analyzing the flow of an isolated liquid plug which is in direct contact with the capillary tube wall. 
When a liquid plug flows in a small-diameter tube $(\mathrm{Bo}<2)$, the capillary forces play a major role and gravity becomes relatively insignificant. In such a case, the interfacial tension between solid-liquid $\left(\sigma_{\mathrm{SL}}\right)$ and solid-gas $\left(\sigma_{\mathrm{SG}}\right)$ should also be taken into account during analysis. An isolated moving liquid plug inside a capillary tube is enclosed by two menisci. During its motion, the dynamic contact angle formed between the flowing liquid meniscus and the solid surface is achieved by a balance between the capillary and the viscous forces. Thus the moving menisci of the liquid plug are characterized by dynamic advancing and receding contact angles, which are different from the static equilibrium contact angle, because of the interplay between surface tension and viscous forces. The relative scaling of these two forces is represented by the capillary number $(\mathrm{Ca}=\mu U / \sigma)$. Rose and Heins (1962) first highlighted the relation between the advancing contact angle and average velocity $U$ of a three-phase contact line. Later, various researchers such as Hoffman (1974), Tanner (1979), and Blake (2006) studied the wetting behavior and dependency of the advancing contact angle on $\mathrm{Ca}$ and the static contact angle. Tripathi et al. (2010) and Shekhavat et al. (2009) previously tried to experimentally understand such phenomenon in oscillating contact line motion in a square capillary tube and presented the hydrodynamic behavior of an oscillating meniscus. The present work is an attempt to analyze the heat transfer in a moving, isolated liquid plug by including the contact angle hysteresis. Two fluids, glycerin and water, have been chosen to get a range of Prandtl number, as well as static wettability characteristics. Further, Taylor flow conditions can occur either as (i) a wet-plug flow regime where a liquid thin film exists at the wall around the bubble (prewetted capillary) and, (ii) a dry-plug flow regime where no such film exists. We focus only on the latter case while the former is intended for future scrutiny.

\section{PROBLEM FORMULATION}

\subsection{Physical Description}

Referring to Figs. 1(b) and 1(c) and Fig. 2(a), a liquid plug of length $L$ is moving inside a previously dry capillary tube of inner diameter $\mathrm{D}$ with a constant average velocity $U_{\text {avg, }}, \theta_{\mathrm{a}}$ and $\theta_{\mathrm{r}}$ being the dynamic advancing and receding contact angles of the interface, respectively. The static contact angle $\theta_{\mathrm{s}}$ is applicable when $U=0$. While moving, the plug is subjected to either a constant temperature or a constant heat flux boundary condition at the capillary tube wall. Because of low Bond number $(\mathrm{Bo}<2)$, the gravity effect is negligible and surface tension effects, in conjunction with viscous forces, play an important role in determining the thermo-hydrodynamic behavior of the plug. 


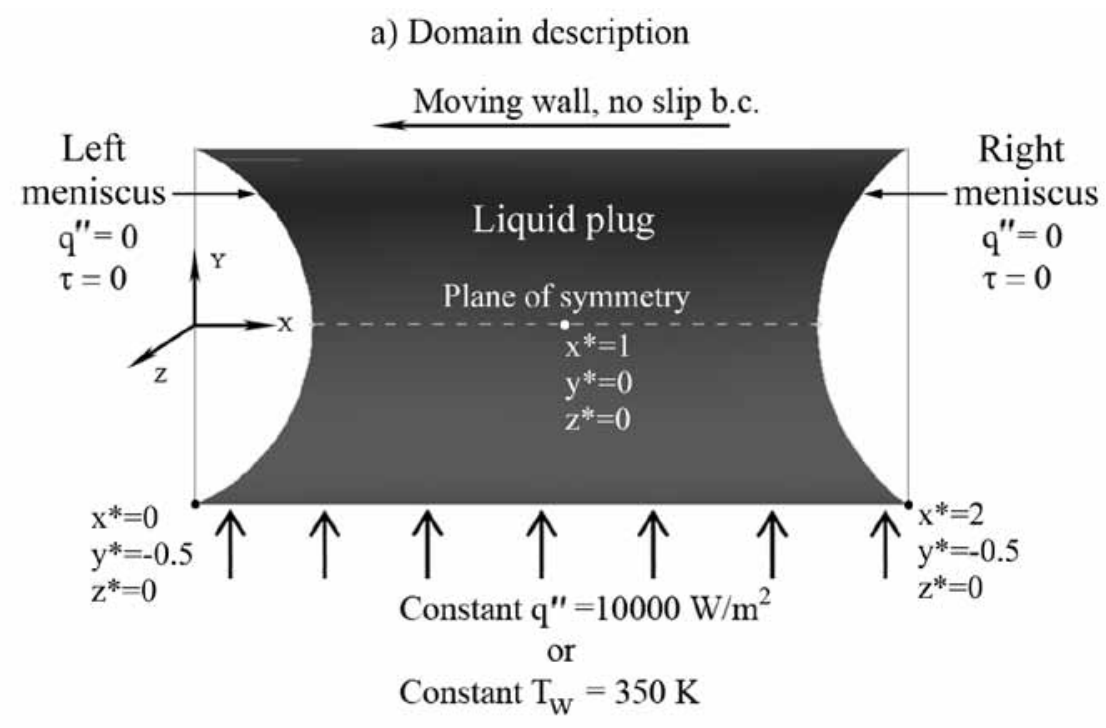

b) Mesh at cross section $z=0$

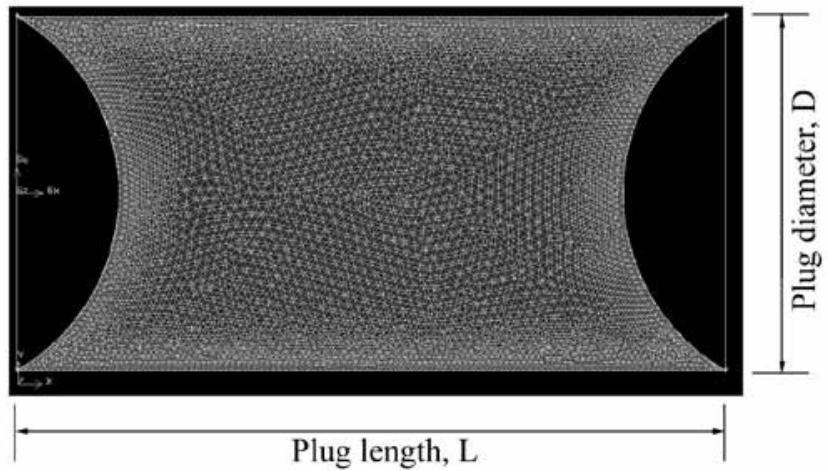

FIG 2: (a) Description of the physical domain of the problem with the applicable boundary conditions used for 3-D simulation of isolated liquid plug flow, and (b) typical mesh structure representing the computational domain.

\subsection{Modeling of Dynamic Contact Angles}

A modified Hoffman-Tanner's law is used to determine the resulting dynamic contact angles when the meniscus moves with a constant velocity $U_{\text {avg. }}$. According to this law, the dynamic contact angle of a moving three-phase contact line is given as (Berthier, 2008)

$$
\theta_{\mathrm{d}}^{3}-\theta_{\mathrm{s}}^{3}+A \mathrm{Ca}
$$


where $\theta_{\mathrm{d}}$ and $\theta_{\mathrm{s}}$ are dynamic and static contact angles, respectively, $\mathrm{Ca}$ is capillary number, and $A$ is a constant whose value depends on the solid-liquid combination. In this study it is taken to be equal to 94 when angles are taken in radians, as suggested by Berthier (2008), who further simplified this model and linearized Eq. (1) by a series expansion, applicable under the assumption of small capillary number $(\sim \mathrm{Ca}<0.1)$. Hence, the dynamic advancing and receding contact angles, $\theta_{\mathrm{a}}$ and $\theta_{\mathrm{r}}$, respectively, are given as

$$
\begin{aligned}
& \theta_{\mathrm{a}}=\theta_{\mathrm{s}}+\frac{A|\mathrm{Ca}|}{3 \theta_{\mathrm{s}}^{2}} \\
& \theta_{\mathrm{r}}=\theta_{\mathrm{s}}-\frac{A|\mathrm{Ca}|}{3 \theta_{\mathrm{s}}^{2}}
\end{aligned}
$$

\subsection{Governing Equations}

The governing equations, i.e., continuity, momentum and energy equations for a Newtonian incompressible fluid with constant properties respectively, can be written as:

$$
\begin{gathered}
\nabla \cdot \vec{V}=0 \\
\rho \frac{D \vec{V}}{D t}=-\nabla p+\mu \nabla^{2} \vec{V} \\
\rho C_{\mathrm{p}} \frac{D T}{D t}=k \nabla^{2} T
\end{gathered}
$$

In the domain of liquid plug, the local Nusselt number at any given location on the tube wall is defined as

$$
\mathrm{Nu}_{x}=\frac{h_{x} D}{k}
$$

where $D$ is the diameter of the tube and the local heat-transfer coefficient $h_{\mathrm{x}}$ is given by

$$
h_{x}=\left.\frac{q^{\prime \prime}}{\left(T_{\mathrm{w}}-T_{\text {avg }}\right)}\right|_{x}
$$

where $q^{\prime \prime}$ is the local wall heat flux, $T_{\mathrm{w}}$ is the local wall temperature, and $T_{\text {avg }}$ is the local mixing temperature of the fluid in the plane perpendicular to the wall at location $x$. In the case of a constant heat flux boundary condition, $q$ " is known 
to us, and $T_{\text {avg }}$ and $T_{\mathrm{w}}$ are obtained from the solution data at the wall. In case of a constant-temperature boundary condition, $T_{\mathrm{w}}$ is known, and $T_{\text {avg }}$ and the ensuing local heat flux are obtained from the solution. An average Nusselt number along the length of the plug is then given by

$$
\mathrm{Nu}_{\text {avg }}=\frac{1}{L} \int_{0}^{L} \mathrm{Nu}_{x} d x
$$

\subsection{Nondimensionalization}

Space variables are nondimensionalized by diameter of the tube $D$. Hence nondimensional parameters are defined as

$$
x^{*}=\frac{x}{D} ; y^{*}=\frac{y}{D} ; z^{*}=\frac{z}{D}
$$

where $x, y$, and $z$ are the spatial coordinates and $x^{*}, y^{*}$, and $z^{*}$ are the nondimensional spatial coordinates. Figure 2(a) shows the nondimensional coordinates of some points. Similarly, velocity is nondimensionalized as

$$
u^{*}=\frac{u}{U_{\mathrm{avg}}} ; v^{*}=\frac{v}{U_{\mathrm{avg}}} ; w^{*}=\frac{w}{U_{\mathrm{avg}}}
$$

where $u, v$, and $w$ are the orthogonal velocity components along the axes, respectively, and $u^{*}, v^{*}$, and $w^{*}$ are the corresponding nondimensional velocities, $U_{\text {avg }}$ being the average velocity of the liquid plug. Time is nondimensionalized based upon the recirculation time scale (time required for a liquid particle to go around the liquid plug once), defined as follows (Thulasidas et al., 1997):

$$
t^{*}=\left(\frac{U_{\mathrm{avg}}}{2 L+D}\right) t
$$

where $L$ is the length of liquid plug along the flow direction and $t$ is the real time in seconds.

\subsection{Computational Simulation Methodology}

The moving frame technique is used to analyze the heat transfer and simulate the flow with moving walls, while keeping the liquid plug stationary. The circular capillary tube wall is given a velocity in the negative $X$ direction while the plug is at rest, as shown in Fig. 2(a). A symmetric boundary condition is used at plane $Z=0$, while a no-slip boundary condition is used at the 
wall. The liquid-gas interface is simulated with zero heat flux and zero shear stress, i.e., a free surface boundary condition. Both constant heat flux $\left(q^{\prime \prime}=\right.$ $\left.10,000 \mathrm{~W} / \mathrm{m}^{2}\right)$ and constant-temperature boundary conditions $\left(T_{\mathrm{w}}=350 \mathrm{~K}\right)$ at the wall have been solved, respectively. Initial temperature of the fluid is taken to be $300 \mathrm{~K}$; fluid thermophysical properties are kept constant. Table 1 gives the range of parameters used for the study. Geometry and mesh is created using Gambit 2.3, with unstructured tetrahedral elements; Fig. 2(b) shows a typical grid structure on the computational domain. The model is solved using an unsteady pressure-based solver in Ansys-Fluent ${ }^{\circledR}$-v12.0 (2009) for water and glycerin, thus giving a wide range of Prandtl number and surface wettability conditions. The PISO algorithm is used for pressure-velocity coupling, while the PRESTO interpolation is used for computing the face pressure. Momentum and energy equations are discretized using a second-order upwind scheme for better accuracy, while temporal discretization is done using a second-order implicit scheme. The Green-Gauss node-based averaging scheme is used to evaluate gradients and derivatives, as recommended by the Ansys-Fluent manual (2009).

TABLE 1: Range of parameters

\begin{tabular}{c|c|c|c|c}
\hline Fluid & Ca & $\mathbf{R e}_{\mathbf{D}}$ & $\mathbf{P r}$ & Bo \\
\hline \multirow{2}{*}{ Water } & \multirow{2}{*}{$10^{-5}-10^{-3}$} & $1.42-142$ & $3.56-7.0$ & 0.737 \\
\cline { 3 - 5 } Glycerin & & $2.5 \times 10^{-6}-2.5 \times 10^{-4}$ & 6780 & 0.883 \\
\hline
\end{tabular}

\section{PROCEDURE}

\subsection{Experimental Investigation of Contact Angle}

To determine the initial equilibrium static contact angle of the working fluids, water and glycerin are carefully introduced in dry capillary tubes so as to produce isolated single plugs, respectively. The capillary tubes are made of Pyrex glass of inner diameter $2.0 \mathrm{~mm}$ and are thoroughly cleaned and rinsed before the experiment. Images are captured using a high-resolution digital camera (Photron ${ }^{\circledR}$-SA3) fitted with a macro lens. Figures 3(a) and 3(b) show the captured images with respective static contact angle for water and glycerin plugs, respectively. Hence, the average static equilibrium contact angle for water is taken to be $67^{\circ}$ and that for glycerin is taken to be $30^{\circ}$, with an uncertainty of $\pm 1^{\circ}$. 


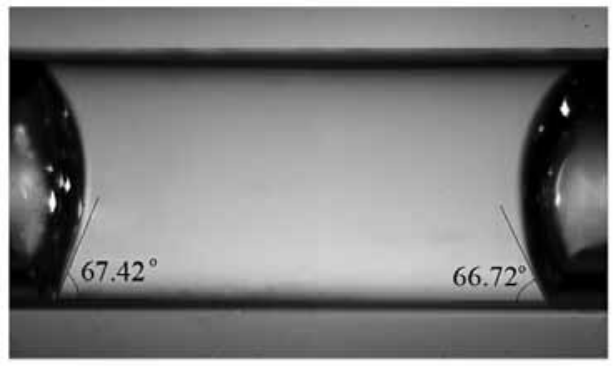

(a) Water plug

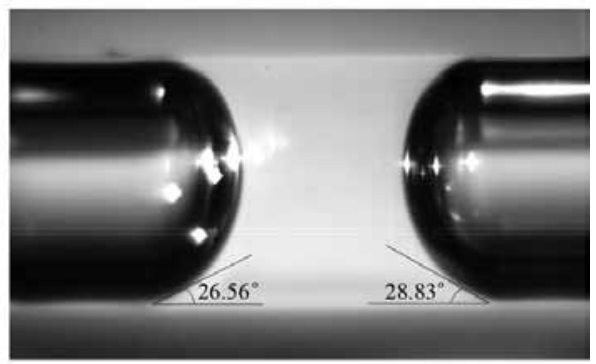

(b) Glycerin plug

FIG 3: Captured digital images of an isolated liquid plug of (a) water and (b) glycerin, placed inside the capillary tube of internal diameter $2.0 \mathrm{~mm}$; also shown are the static contact angle made by the respective plugs.

\subsection{Numerical Simulations}

Three-dimensional (3-D) numerical simulations are carried out using the procedure described above for different $L / D$ ratios of the liquid plugs, i.e., $L / D=1,2$, and 4 and for different capillary numbers, $\mathrm{Ca}=10^{-5}, 10^{-4}$, and $10^{-3}$, for water and glycerin liquid plugs, at constant temperature and constant heat flux boundary conditions, respectively. A grid independence test is carried out for four different grid element sizes at $\mathrm{Ca}=10^{-3}$ and $L / D=2$, taking glycerin as the working fluid. Figures 4(a) and 4(b) show the grid independence test, and Fig. 4(c) shows the validation test against fully developed Poiseuille flow. In Fig. 4(a), nondimensional centerline velocity is plotted against distance from the tube entrance. It is clear that the results are independent of the grid after a starting element size of 0.04 . Hence, all other simulations are carried out taking this as starting smallest element size. Also, Fig. 4(b) shows the velocity profile at the center of the plug at a symmetric plane $\left(z^{*}=0\right)$ at different element size and compares them against a fully developed parabolic Poiseuille flow velocity profile. It is clear that the velocity profile at element size 0.04 matches with the parabolic profile. To validate the present model, a test is carried out against fully developed Poiseuille flow by extending the length of the plug to $L / D=25$. Figure 4(c) shows the results for this simulation. The maximum error between the theoretical results and simulation results is $0.05 \%$ at the point of maximum velocity, which is considered to be satisfactory.

\section{RESULTS AND DISCUSSION}

As mentioned earlier, simulations are carried out for water and glycerin working fluid and for a range of capillary numbers and $L / D$ ratios. The transient form of the governing equations are cast and are solved with a time step size $0.0015 \mathrm{~s}$, until steady 

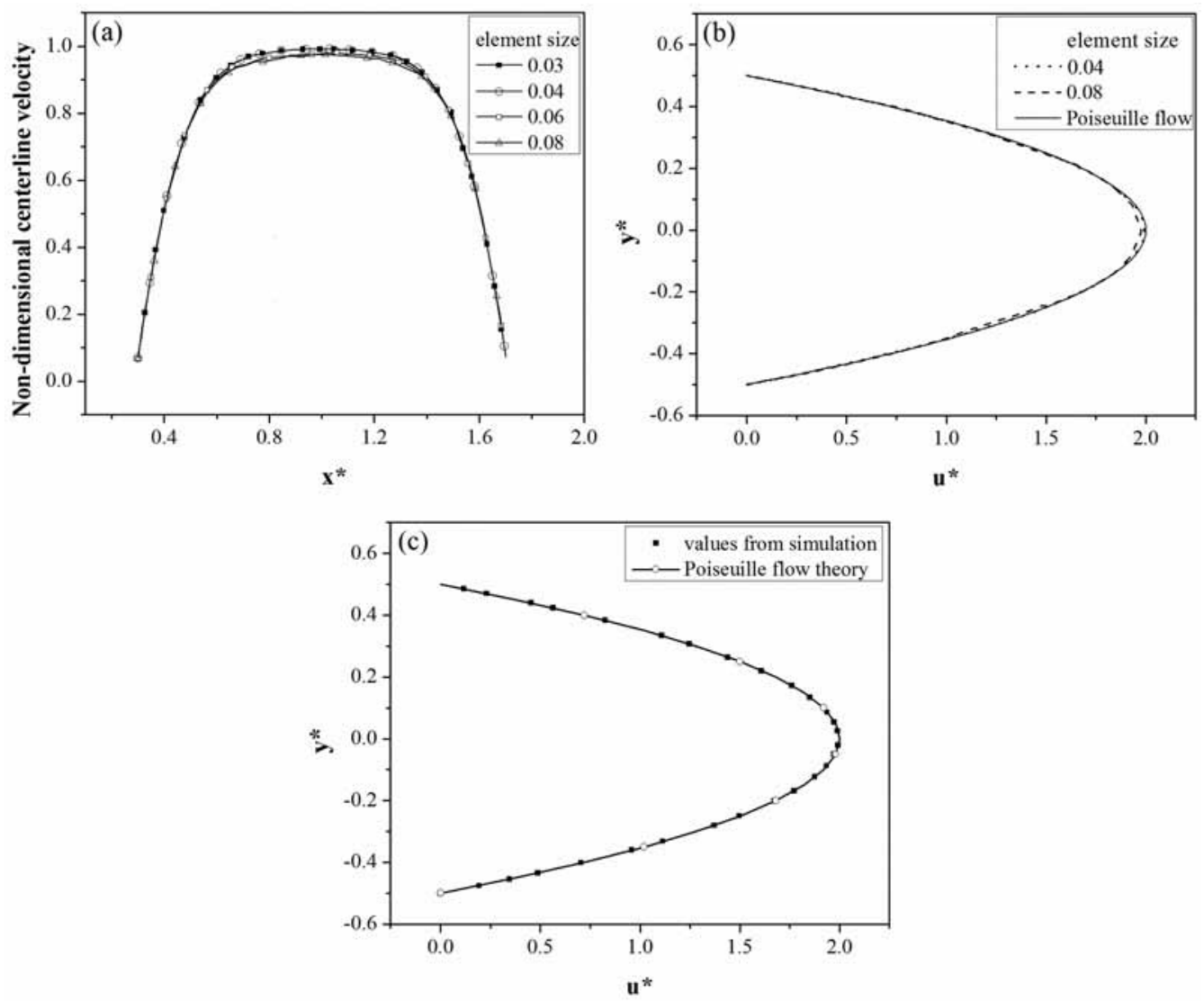

FIG 4: Details of the grid independence test for glycerin plug at $L / D=2$ and $\mathrm{Ca}=10^{-3}$ : (a) nondimensional centerline velocity and (b) nondimensional $u$-velocity profile at the center of the plug $\left(x^{*}=1\right)$. (c) Validation against fully developed Poiseuille flow in a circular tube at $\operatorname{Re}=140$.

state is reached. Though many studies have been carried out to understand plug flow systems, a full 3-D description of a velocity field near the interface is not available in the literature. Because of the limitations of the PIV method, only velocities in two directions are obtained through experiments. In the present study, we have carried out 3-D simulations in order to get the full velocity field near the interface. It has been found that flow is multidimensional near the interface with all the three velocity components existing near it, while far from the interface, flow is essentially one dimensional if it is completely developed or two dimensional in the case of developing flow. In the following section, we discuss the variation of velocity, streamlines, and heat transfer in the test cases. 


\subsection{Velocity Field}

Figures 5(a)-5(c) show the steady-state velocity profiles in $X, Y$, and $Z$ directions at different axial locations for a water plug at $\mathrm{Ca}=10^{-3}$ and $L / D=2$ [refer to Fig. 2(a)], wherein the nondimensional coordinates of some corner points have also been depicted for clarity. Figure 5(d) shows the $X$-direction velocity $\left(u^{*}\right)$ developed on the center plane of the liquid plug $\left(x^{*}=1\right.$ and $\left.z^{*}=0\right)$ with time. The plug started from rest, and with time the center of the slug attained the steadystate parabolic profile, as seen. Figure 5(a) gives the steady-state velocity profile in $X$ direction $\left(u^{*}\right)$, Fig. 5(b) gives the profile in $Y$ direction $\left(v^{*}\right)$, and Fig. 5(c) gives the velocity profile in $Z$ direction ( $\left.w^{*}\right)$. From Fig. 5(a) it is clear that near the interface, velocity is maximum before the axis, i.e., $y^{*}=0$. There is a dip in the velocity near the interface, the interface being located at $x^{*}=0.12$. Away from the interface, $u^{*}$ starts flattening and developing afterward. At $x^{*}=1$, the velocity profile takes the shape of a fully developed parabolic profile, as in the case of Poiseuille flow. Hence, it can be deduced that if the length of the plug is sufficient enough $(L / D>\sim 2)$, then velocity will tend to be fully developed in some region in the middle of the plug. As mentioned by Thulasidas et al. (1997), the flow profile in the developed region inside the plug is given by

$$
u(r)+\frac{2 U_{\mathrm{b}}}{\psi}\left(1-\frac{r^{2}}{R^{2}}\right)
$$

where $u(r)$ is the velocity in axial direction, $U_{\mathrm{b}}$ is the bubble velocity, $\Psi$ is the dimensionless bubble velocity $\left(\Psi=U_{\mathrm{b}} / J, J\right.$ is the total volumetric flux across the capillary), $r$ is the radial distance from the axis, and $R$ is the radius of the capillary tube. In the case of a dry-plug flow regime, liquid and bubble move with the same velocity because no thin film exists at the wall and thus $\Psi$ is equal to 1 and $U_{\mathrm{b}}=J=U_{\text {avg }}$; hence, the above equation gets reduced to

$$
u(r)=2 U_{\text {avg }}\left(1-\frac{r^{2}}{R^{2}}\right)
$$

At the plane of symmetry $\left(z^{*}=0\right), y=r$, and hence, we can write

$$
u(y)+2 U_{\text {avg }}\left(1 \frac{y^{2}}{R^{2}}\right)
$$

It is also clear that the $u$-velocity profile starts developing after the interface and at $x^{*}=1$, flow gets fully developed and takes the shape of a parabolic velocity profile. 

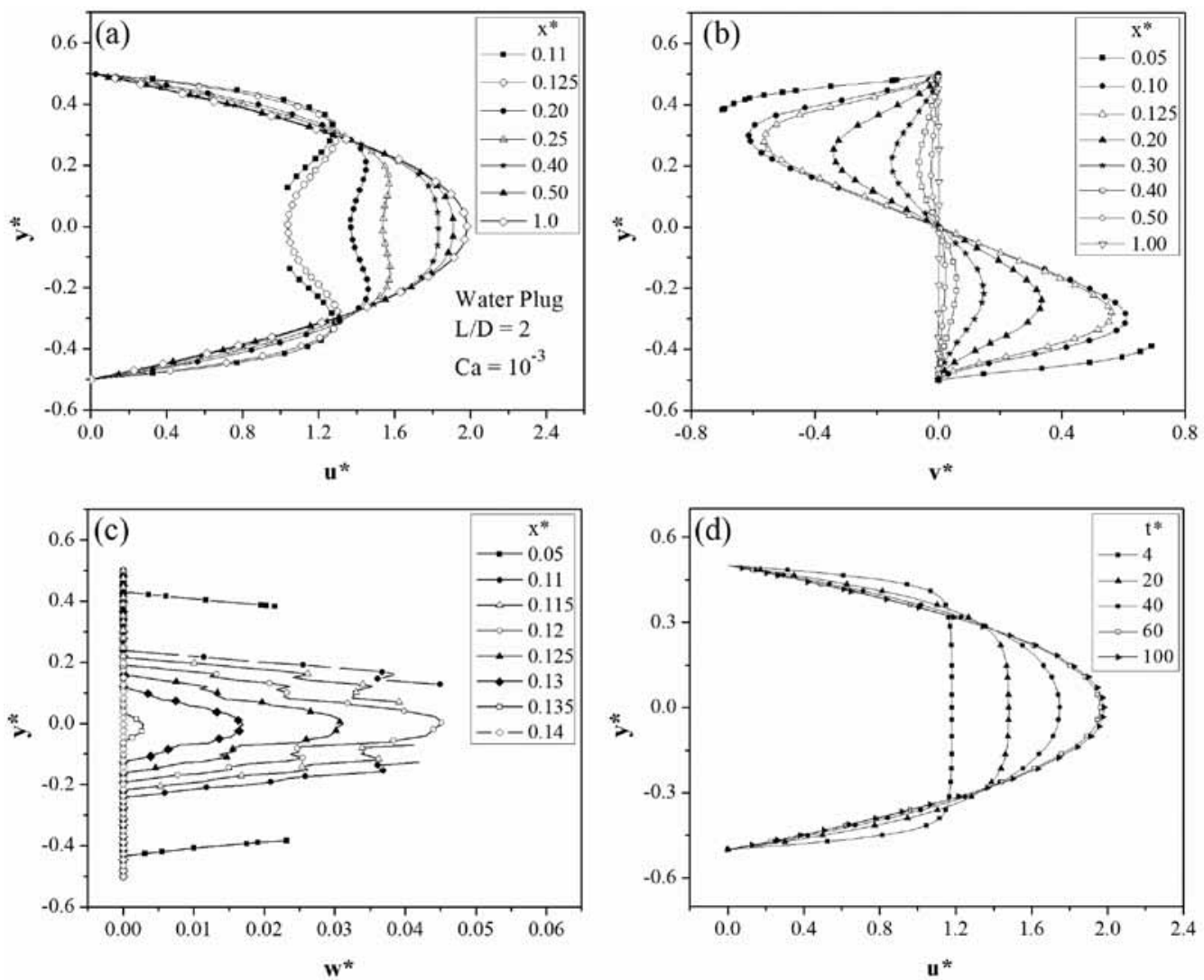

FIG 5: Steady-state velocity profiles at different cross sections on the symmetric plane $\left(z^{*}=0\right)$ for water plug at $L / D=2$ and $\mathrm{Ca}=10^{-3}$ : (a) u-velocity profile, (b) $v$-velocity profile, (c) $w$-velocity profile, and (d) temporal development of the velocity profile at the center of the plug $\left(x^{*}=1\right.$ and $\left.z^{*}=0\right)$.

Figure $5(\mathrm{~b})$ gives the nondimensional $v$-velocity profile at different cross sections at the symmetric plane $\left(z^{*}=0\right)$ of the plug. Velocity is at its maximum near the interface, and then starts decreasing with $x^{*}$. At the center of the plug $\left(x^{*}=1\right)$, velocity is essentially one dimensional and the $y$ component of velocity becomes zero in the center. Figure 5(c) shows the $z$ component of velocity in the liquid plug. This clearly indicates the presence of 3-D flow near the interface. The 3-D flow near the interface has been mentioned by some authors, but the velocity profile has not been explicitly reported in the open literature. The interface is located at $x^{*}=0.12$ and $w^{*}$ is maximum at this interface. On going away from the interface, it starts decreasing rapidly and becomes zero at $x^{*}=0.14$. Hence, 
later on, flow can be assumed to be two-dimensional (as flow is still developing and the $y$ component of velocity exists). But near the interface, the flow is clearly $3-\mathrm{D}$, with a strong presence of $z$ component of velocity. However, if we compare the order of the velocity profiles in $X, Y$, and $Z$ directions near the interface, the $\mathrm{u}$ velocity is of the order of $1.2, v$ velocity is of the order of 0.6 , while $w$ velocity is of the order of $0.05,10$ times smaller than the $v$-velocity profile. Hence, though the flow is 3-D near the interface, yet the $Z$ component of velocity is not very significant. The same order-of-magnitude behavior is also observed for a glycerin plug under the similar conditions.

Figure 6 compares the velocity profiles at the front of the interface $\left(x^{*}=0.13\right)$ for wet-plug flow as given by He et al. (2010) and for the dry-plug flow from the present study. Since not many studies are available for the dry-plug flow regime, the velocity profile from wet-plug flow is compared to draw an analogy. From Fig. 6, it is clear that the shape of the velocity profile is qualitatively quite similar for the dry-plug and the wet-plug flow regime, except for the fact that the velocity peak for the wetplug flow regime is higher than that of the dry-plug flow. This is expected, as in the wet-plug flow, the interface has less cross-sectional area compared to that in the dryplug flow regime because of the existence of a thin film around the bubble and wall. Because of this thin film, the shape of the interface is more curved in the wet-plug flow compared to that in dry-plug flow, hence giving rise to higher peak velocity. For the same reason, velocity at $y^{*}=0$ is the same for both cases, as the curvature of the interface would be the same at this point for both types of flows.

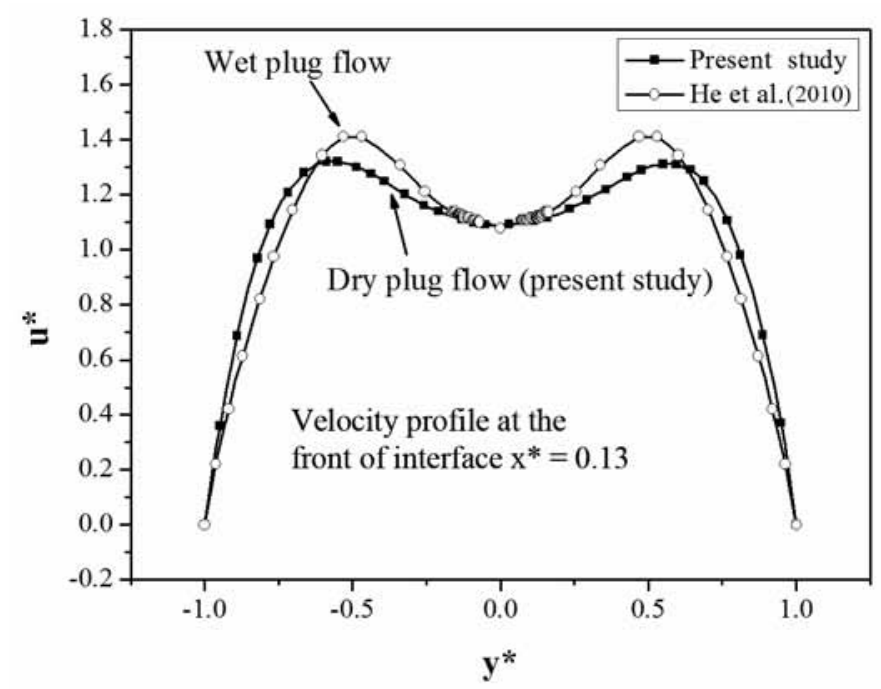

FIG 6: Comparison of velocity profiles in front of the interface, at location $x^{*}=0.13$. 
For a shorter plug $(L / D=1)$, the velocity profile could not develop fully and remains in the developing state. This is also clear from Fig. 5(a), as velocity is fully developed at $x^{*}=1$, which is the center of the plug for $L / D=2$. For $L / D=1$, the center of the plug is at $x^{*}=0.5$, and flow remains underdeveloped in this case. A better idea of velocity distribution in the plug can be obtained by analyzing the streamlines. Figures 7(a) and 7(b) show the streamlines in the frame of reference moving with the wall for $\mathrm{Ca}=10^{-3}$ for water and glycerin cases, respectively.

Figure 7(a) shows the streamlines for $L / D=1$ and $\mathrm{Ca}=10^{-3}$ for the water plug at the plane of symmetry, i.e., $z^{*}=0$. The streamlines for this case are curved all over the plug, showing the developing nature of flow for such short plugs. Because of the proximity of interfaces, there is only one vortex in this case, in the upper half of the plane, which is affected by both the interfaces. Strong internal circulations, evident from this simulation, are the main cause of increased heat transfer for this short plug, as will be discussed later. The fluid in the center moves faster compared to fluid near the wall, and impinges the interface. Because of the immiscibility of two phases (gas-liquid), this liquid phase reverses its direction, giving rise to the strong internal circulations. On the other hand, for $L / D=2$ and 4 , flow becomes fully developed in the center section of the plug and hence streamlines become near parallel straight lines in the center, while remaining curved near the interface because of the 3-D nature of the flow. Another important point to observe from these two cases is the distinct formation of two vortices in the latter two cases. This is again a direct consequence of length of the plug. For higher plug length, interfaces do not interact directly with each other and hence two separate vortices are formed, clearly observable from Figs. 7(a), (ii) and (iii). As the rear meniscus has a larger curvature compared to that of the advancing front meniscus, hence the rear vortex is stronger compared to that of the front vortex. This causes uneven local heat transfer, as discussed later. For higher $L / D$, streamlines are essentially parallel straight lines with poor vortex strength. Hence, it reduces the overall heat transfer too. A qualitatively similar pattern was observed by Thulasidas et al. (1997) and He et al. (2010) within the reference frame of a moving bubble for wet-plug flow also.

Figure $7(\mathrm{~b})$ represents the streamlines for a glycerin plug at $\mathrm{Ca}=10^{-3}$. The noticeable difference between the flow field of water and the glycerin plug is the number of vortices in the two cases. While the former had two vortices at $L / D=2$, the latter has only one vortex due to its higher viscosity. This is another reason for low heat transfer in glycerin compared to that in water. Table 2 gives information on the location of the center of vortices for the water case for different $L / D$ ratios and compares its radial location, as given by Thulasidas et al. (1997). 
(i) $\mathrm{L} / \mathrm{D}=1$
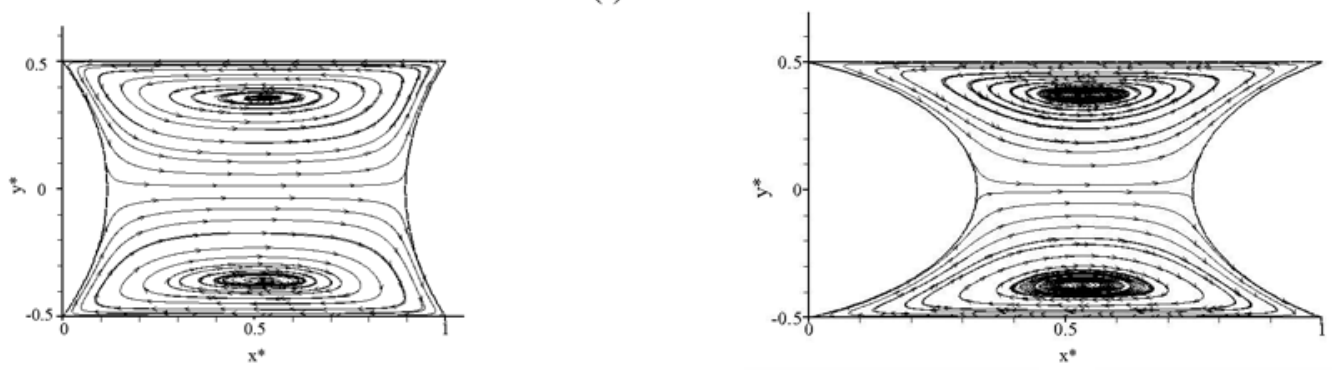

(ii) $\mathrm{L} / \mathrm{D}=2$
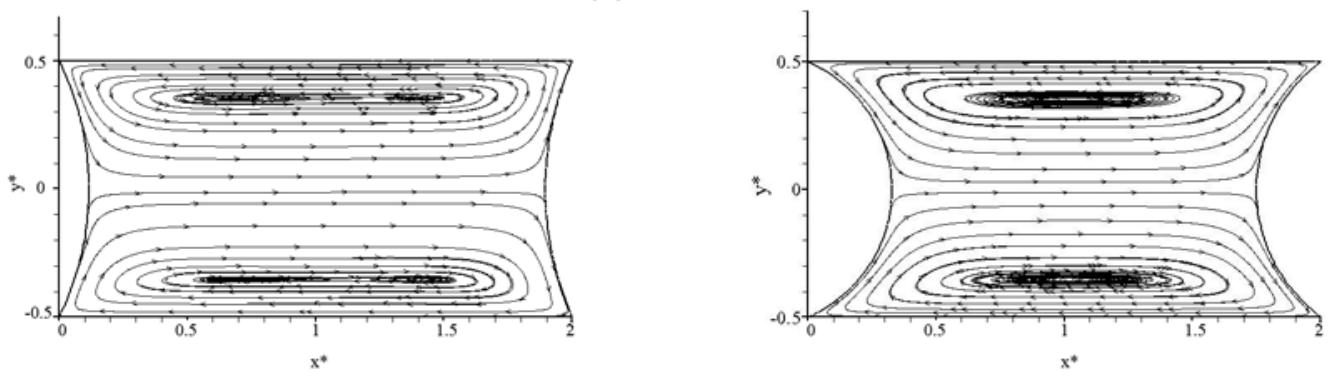

(iii) $\mathrm{L} / \mathrm{D}=4$

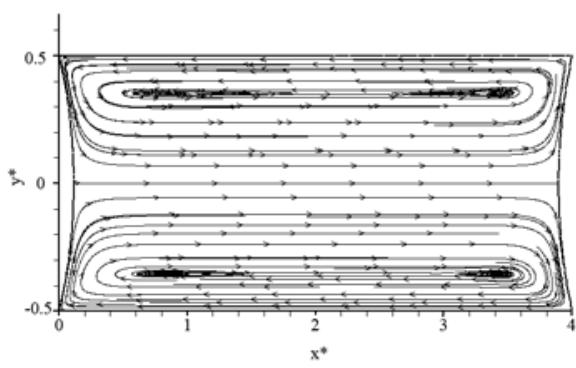

(a) Water

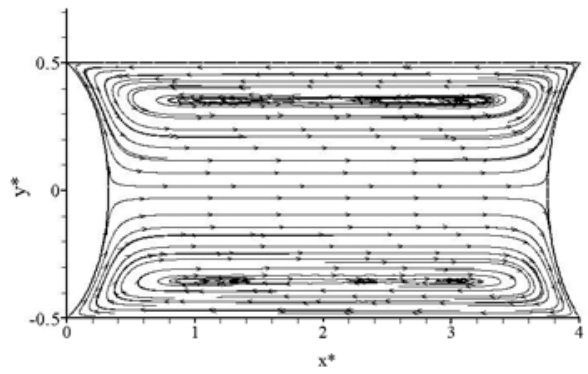

(b) Glycerin

FIG 7: (a) Steady-state streamlines at the symmetric plane $\left(z^{*}=0\right)$ at $\mathrm{Ca}=10^{-3}$ and varying $L / D$ ratio (a) inside a water plug and (b) inside a glycerin plug. Note the difference in the linear scales used for the two fluids for enhancing clarity of presentation.

The radial location by Thulasidas et al. (1997) is given only for developed velocity profile in wet-plug flow which in this case is for $L / D=2$ and 4 . The present results are very close to the theoretical values given by Thulasidas et al. (1997). There is a slight difference between the simulation values and theoretical values. This difference might be because of contact angle hysteresis which affects the position of rear and front vortex. Another interesting feature to note is the shifting of vortex towards the tube axis and away from interface with increasing $L / D$. 
TABLE 2: Position of vortex in the water plug

\begin{tabular}{c|c|c|c}
\hline $\boldsymbol{L} / \boldsymbol{D}$ & $\boldsymbol{x}^{*}$ (axial location) & $\boldsymbol{y}^{*}$ (radial location) & $\begin{array}{c}\text { Radial location } \boldsymbol{y}^{*} \text { ) } \\
\text { (Thulasidas } \text { et al., 1997) }\end{array}$ \\
\hline 1 & 0.5283 & 0.3583 & - \\
\hline 2 & 0.6528 & 0.3538 & 0.3535 \\
\hline 4 & 0.8093 & 0.3530 & 0.3535 \\
\hline
\end{tabular}

\subsection{Heat Transfer}

Figures 8 and 9 show the temporal variation of average Nusselt number (averaged over the length of the plug) for water and glycerin plugs, respectively, with $L / D=2.0, \mathrm{Ca}=10^{-3}$, under constant heat flux and constant-temperature boundary conditions, respectively. As noted earlier, the plug recirculation time scale, $(2 L+D) / U_{\text {avg }}$ is used to define the nondimensional time. Two separate cases are reported for comparison: (i) taking dynamic contact angle variations, as per Eq. (2) and, (ii) fixed/invariant dynamic contact angles, equal to the respective static contact angles, irrespective of the plug velocity.

Several important aspects are noted from Figs. 8 and 9. First, steady-state $\mathrm{Nu}_{\text {avg }}$ is attained very early in the case of the glycerin plug. Another notable feature is the initial fluctuations observed in the $\mathrm{Nu}_{\text {avg }}$ in case of the water plug; these are absent in the glycerin system. In addition to the fact that glycerin has a very high Prandtl number, its flow Reynolds number is extremely low as compared to the water plug, for the same capillary numbers, as seen in Table 1; this explains the initial transient behavior of the glycerin plug. (It must be noted that the effect of Prandtl number on the heat transfer is only valuable while the capillary number for both these cases is kept constant. Different conclusions will be found when comparing heat transfer, keeping the Reynolds number constant.) In the case of water, the initial upward increase in $\mathrm{Nu}_{\text {avg }}$ is attributed to the rapid motion of the cold fluid in the central core of the plug, which comes in contact with the tube wall because of greater propensity in the frequency of internal circulations that are present in the water plug. Thus $\mathrm{Nu}_{\text {avg }}$ reaches a maximum and then starts declining as the fluid that has previously been heated completes its one circulation cycle and again comes in contact with the wall. The cycle keeps on repeating with decreased amplitude. After some time, an asymptotic steady state is reached and there are no more oscillations in $\mathrm{Nu}_{\text {avg }}$. As explained earlier, such fluctuations are absent in the glycerin plug. Variations in fluctuations in Nusselt number in water and glycerin fluid were also observed by Walsh et al. 

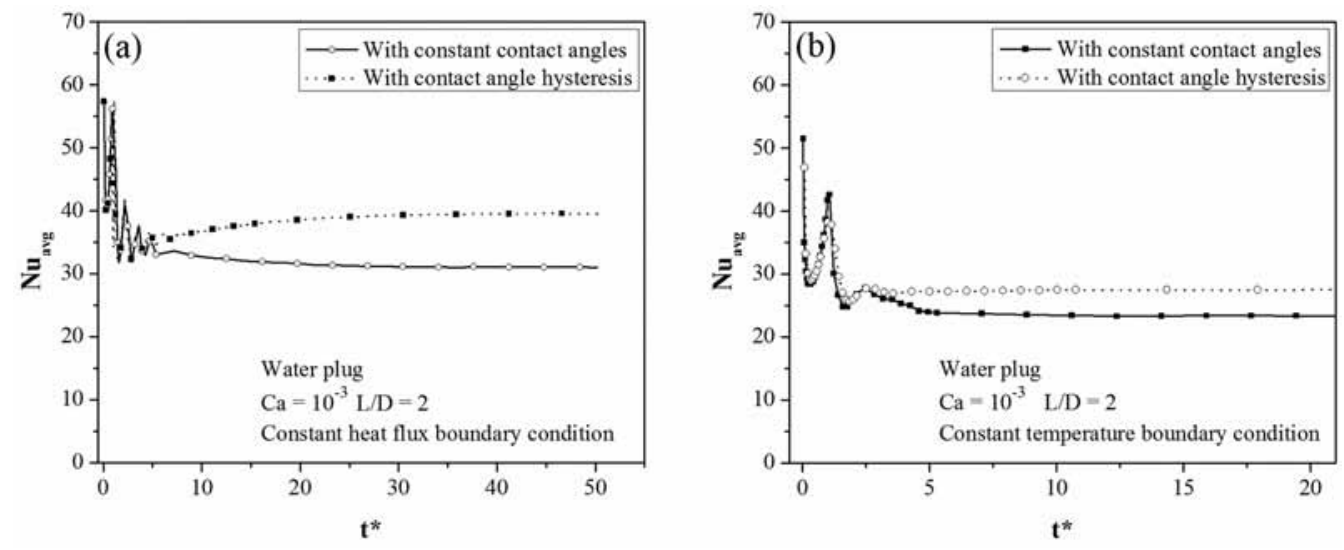

FIG 8: Temporal variation of average Nusselt number $\mathrm{Nu}_{\mathrm{avg}}$ for water plug with constant contact angles and contact angle hysteresis at $\mathrm{Ca}=10^{-3}$ and $L / D=2$ for (a) constant heat flux boundary conditions and (b) constant-temperature boundary conditions. An increase of 33\% in Nusselt number is observed in the case of an isolated plug with contact angle hysteresis under constant heat flux conditions, while it is $28 \%$ under constant-temperature boundary conditions.

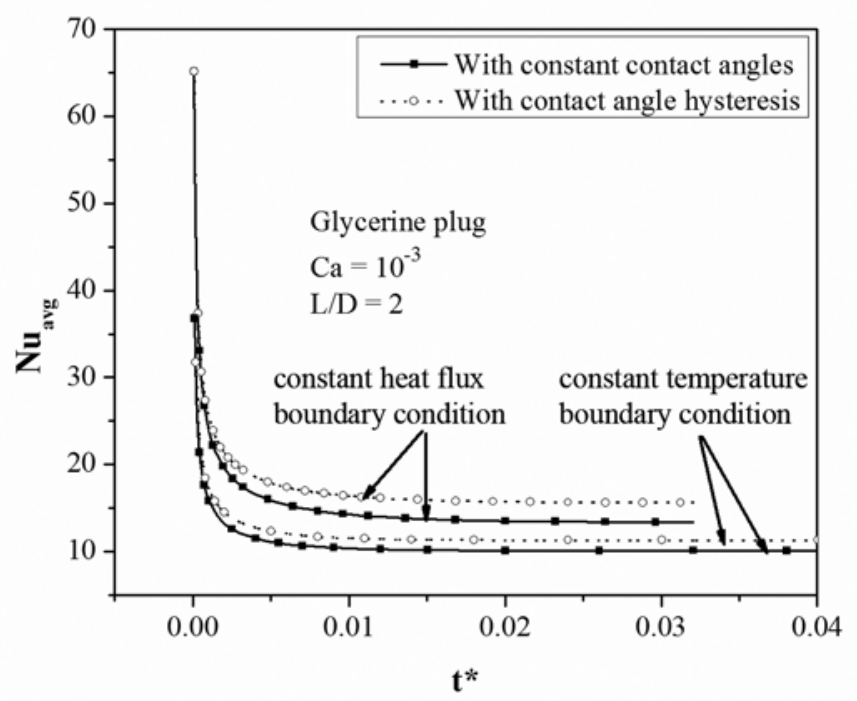

FIG 9: Temporal variation of average Nusselt number $\left(\mathrm{Nu}_{\text {avg }}\right)$ for a glycerin plug with constant contact angles and contact angle hysteresis at $\mathrm{Ca}=10^{-3}$ and $L / D=2$. An increase of $16 \%$ in Nusselt number is observed in the case of contact angle hysteresis under constant heat flux boundary conditions, while this value is $12 \%$ under constanttemperature boundary conditions. 
(2011); however, a satisfactory explanation was not provided in their research work. Finally, when comparing the two cases on the basis of dynamic contact angle hysteresis, it is noted that an increase of about $16 \%$ in the steady-state $\mathrm{Nu}_{\text {avg }}$ is observed in the case of the glycerin plug, while for the water plug, this value is approximately $33 \%$.

For a better understanding of the heat-transfer mechanism in both the plugs, temperature contours are drawn in Figs. 10 and 11 for water and glycerin liquid plugs, respectively, at different times for $\mathrm{Ca}=10^{-3}$ and $L / D=2$. Because of the lower Prandtl number of water as compared to glycerin, a thermal boundary layer develops quite inline to the velocity boundary layer. One complete circulation cycle is shown in Fig. 10 for the water plug. Temperature starts rising in the direction of velocity. As the circulation propagates, hotter liquid near the wall is brought to the center while colder liquid goes toward the wall. After a complete circulation, fluid in the core of the plug is hotter compared to the fluid near the wall. Because of this reason, $\mathrm{Nu}_{\mathrm{avg}}$ experiences oscillations. When one complete cycle is over, it again starts increasing with less amplitude and finally, a steady state is eventually attained.

Figure 11 shows the temperature contour for a glycerin plug at different time steps. Because of the higher Prandtl number, heat transfer mostly takes place through diffusion. Though internal circulations are present in the plug, heat is not carried away by them and hence the increase in Nusselt number is less compared to that of water. Because of one-dimensional heat transfer, no oscillations in $\mathrm{Nu}_{\text {avg }}$ are observed in this case, and after an initial high value of Nusselt number, soon a steady state is reached. Another important reason for this diffusiondominant heat transfer in glycerin is the low Reynolds number in the present case (Table 1). However, if the Reynolds number for both the fluids is kept identical, then the capillary number would be extremely high for glycerin (because of its high viscosity). In this case, the linearized Hoffman-Tanner's law would not be applicable and the present analysis cannot be applied.

Figure 12 shows the steady-state temperature contours for water $(\operatorname{Pr}=7)$, gas-oil $(\operatorname{Pr}=50)$, ethylene-glycol $(\operatorname{Pr}=150)$, and glycerin $(\operatorname{Pr}=6780)$ for $L / D$ $=2$ and $\mathrm{Ca}=10^{-3}$ under constant heat flux boundary conditions. As the Prandtl number increases, momentum diffusion dominates thermal diffusion. Hence, at low $\mathrm{Pr}$, as in water, substantial heat diffusion along with fluid recirculation can be seen, as shown in Fig. 12(a); as Pr increases, the tendency of such a phenomenon keeps on decreasing. At $\operatorname{Pr}=150$, the effect of recirculation is reduced [Figs. 12(b) and 12(c)] and at very high $\operatorname{Pr}(\operatorname{Pr}=6780)$, the effect of recirculation is not felt and heat propagates in almost one dimension [Fig. 12 (d)]. 
Temperature (K)

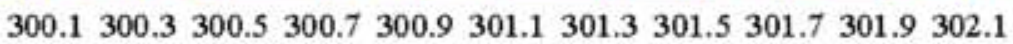

(a)

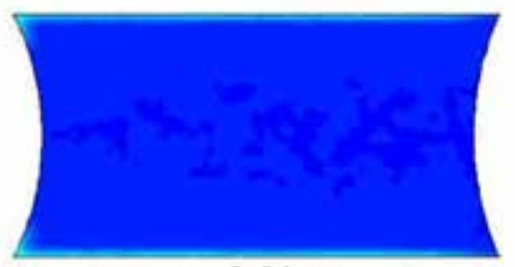

$\mathrm{t}=0.01 \mathrm{sec}$

$t^{*}=0.0717$

(b)

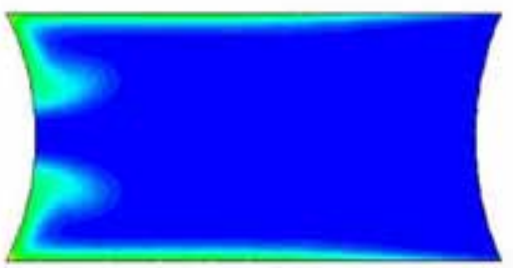

$\mathrm{t}=0.04 \mathrm{sec}$

$t^{*}=0.2868$

(c)

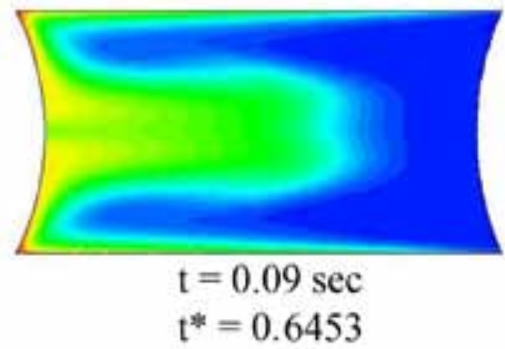

(d)

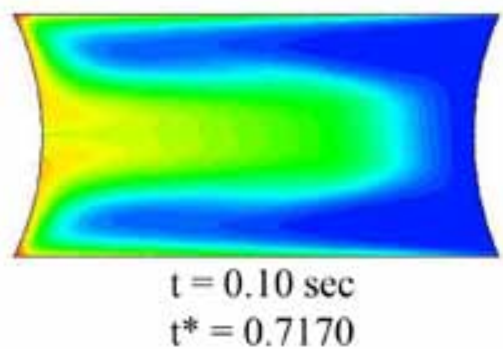

(e)

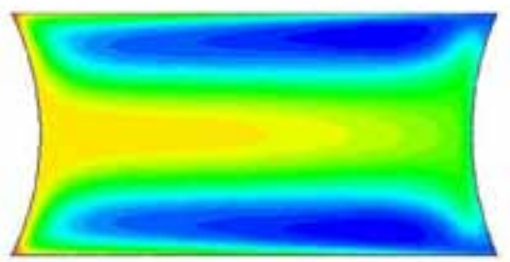

$\mathrm{t}=0.13 \mathrm{sec}$

$\mathrm{t}^{*}=0.9321$

(f)

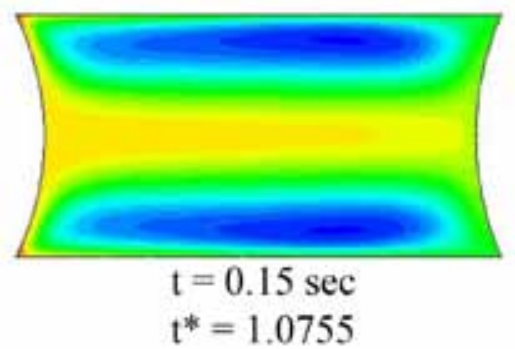

FIG 10: Temporal variation of temperature contour for water plug at $\mathrm{Ca}=10^{-3}$ and $L / D=2$. Strong presence of internal circulation and associated heat transfer is observed, causing the oscillations in Nusselt number in the initial transient phase.

To explain the mechanism of increased heat transfer in the case of contact angle hysteresis, $Z$-vorticity contours and corresponding streamlines are drawn at steady state for both cases for water plug, viz., with constant contact angles and with contact angle hysteresis (Fig. 13). Near-wall vorticity reflects the magnitude of the shear stress, which in turn, scales with the Nusselt number. While the general vorticity behavior for both cases is quite similar, the near-wall vorticity distribution for the case with contact angle hysteresis is indeed higher than its counterpart. This suggests higher near-wall transport, partly explaining the 
Temperature (K)

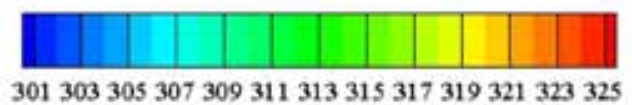

(a)

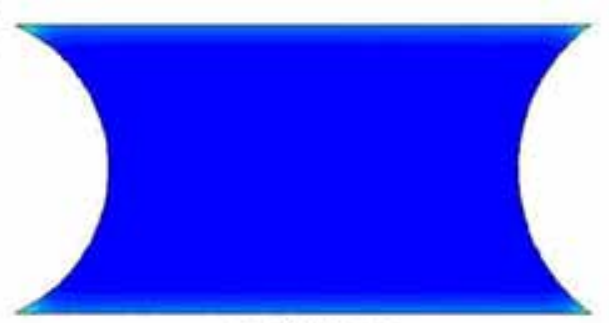

$\mathrm{t}=0.1 \mathrm{sec}$

$\mathrm{t}^{*}=8.01 \mathrm{e}-4$

(b)

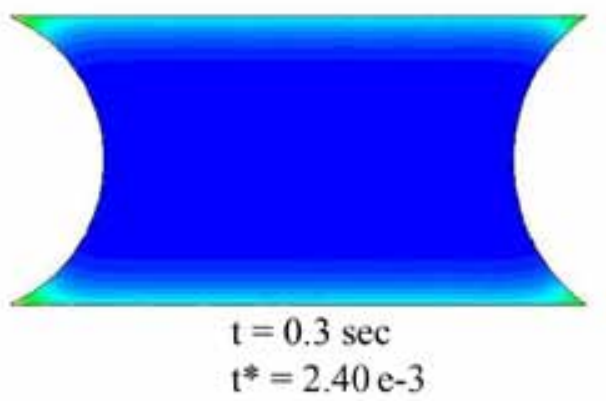

(c)

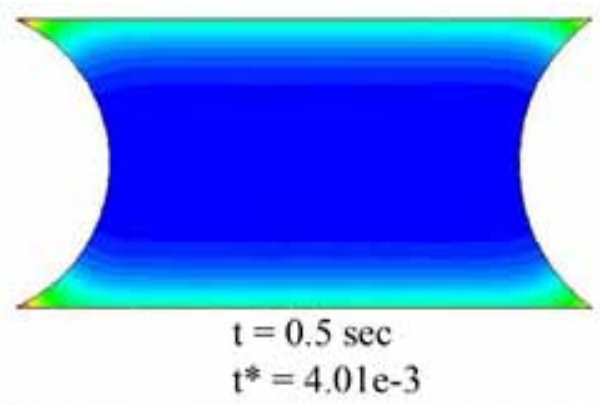

(d)

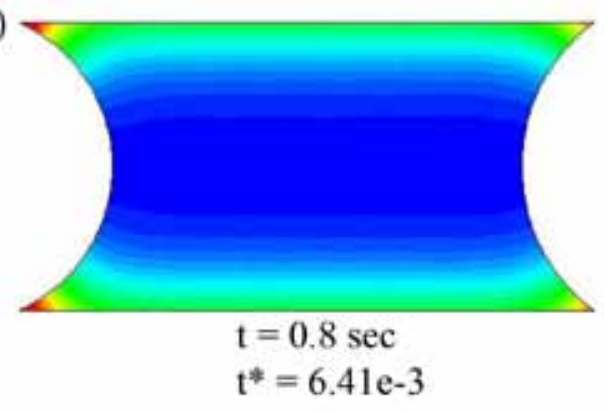

(e)

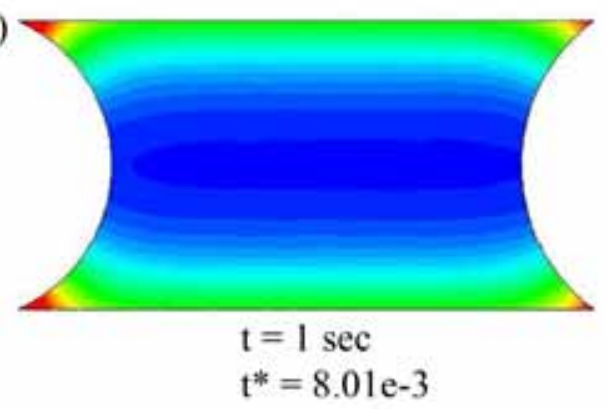

FIG 11: Temporal variation of temperature contour for glycerin plug at $\mathrm{Ca}=10^{-3}$ and $L / D=2$. In this case, heat transfer is one dimensional and is not strongly affected by the flow circulations.

enhanced heat-transfer behavior. Because of the contact angle hysteresis, the rear interface is more curved compared to that of the front interface. This causes stronger circulations and high vortex strength near the rear end. In the case of constant contact angles, these vortices are symmetric and of poor strength compared to those of the latter case.

Figure 14(i) shows the variation of $\mathrm{Nu}_{\mathrm{avg}}$ with $\mathrm{Ca}$, for $L / D=2$, under constantwall-temperature boundary conditions for water and glycerin plugs, respectively. It is clear from these results that at a constant $L / D$ ratio, $\mathrm{Nu}_{\text {avg }}$ increases with $\mathrm{Ca}$ for both the liquids, though this increase is greater in water compared to glycerin. 


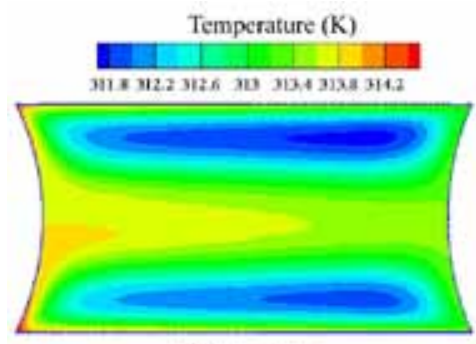

(a) Water, $\mathrm{P}_{1}=7$

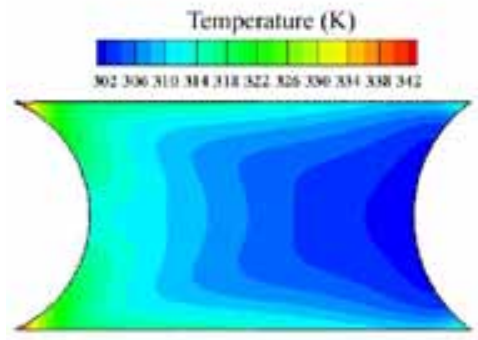

(c) ellyylenc-gly

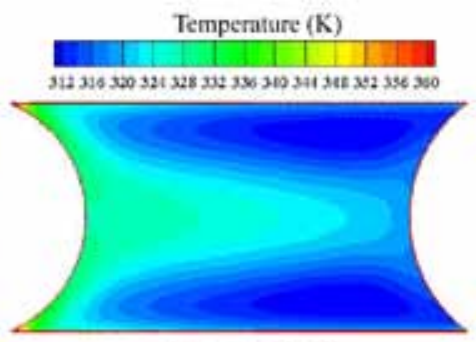

(b) gas - ili, $\mathrm{Pr}=50$

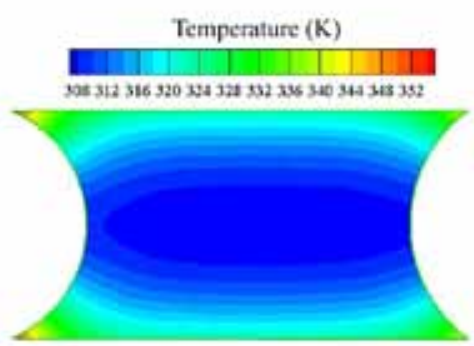

(d) glyecrias, $\mathrm{Pr}-6780$

FIG 12: Steady-state temperature contours for (a) water $(\operatorname{Pr}=7)$, (b) gas-oil $(\operatorname{Pr}=50)$, (c) ethylene-glycol $(\operatorname{Pr}=150)$, and $(d)$ glycerin $(\operatorname{Pr}=6780)$. All cases have been simulated under constant heat flux boundary conditions, with $L / D=2$ and $\mathrm{Ca}=10^{-3}$.

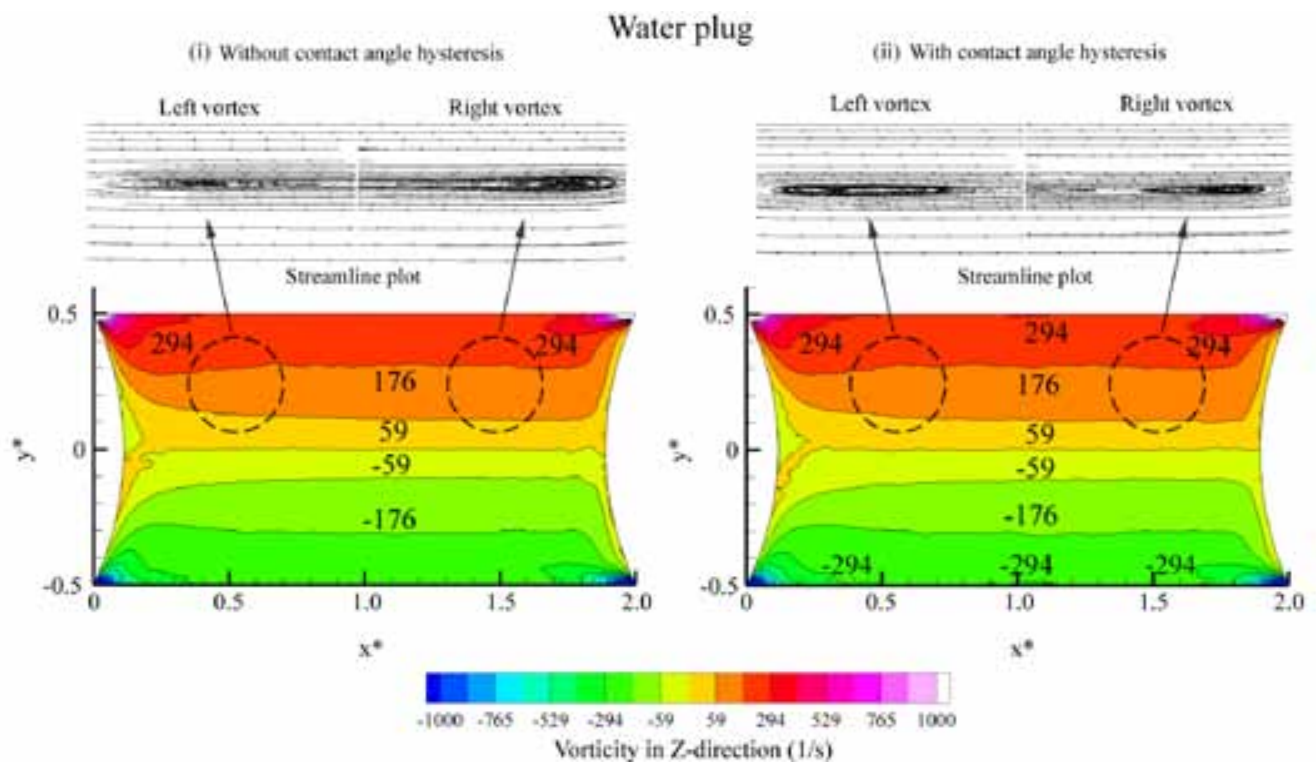

FIG 13: (a) Steady-state vorticity contours and corresponding streamline pattern near the vortices formed inside the liquid plug at $\mathrm{Ca}=10^{-3}$ and $L / D=2$ for a water plug. For both cases: (i) liquid plug with constant contact angles while in motion, and (ii) liquid plug having dynamic contact angle hysteresis. 
Because of high momentum diffusivity, there is no significant effect of increased velocity in the case of glycerin and convection is more or less not much affected. This can also be explained on the basis of temperature contours, as explained earlier. Since heat transfer takes place mostly through diffusion in glycerin plugs, hence an increase in velocity (or $\mathrm{Ca}$ ) does not have much effect on heat transfer. In the case of water, increase in $\mathrm{Ca}$, and thus velocity, has a significant impact on $\mathrm{Nu}_{\text {avg }}$ and an increment of around $50 \%$ is observed. Thus Ca plays an important role in determining the effective heat-transfer coefficient in the case of lowPrandtl-number fluids.

Figure 14(ii) shows variation of $\mathrm{Nu}_{\text {avg }}$ with $L / D$ ratio at $\mathrm{Ca}=0.001$ for a water and a glycerin plug, respectively, under a constant-wall-temperature case. The most important feature to be noted from the graph is that the heat-transfer coefficient increases as the length of the plug decreases (we have not checked whether this is monotonous). A higher heat-transfer coefficient in the case of low aspect ratio $(L / D)$ is because of the decrease in the recirculation time scale, as a result of which, relatively cooler fluid from inside the droplet core is able to reach the walls at a faster rate. This is also clear from Figs. 7(a) and 7(b). In the case of smaller plugs, convective currents are more dominant and internal circulations get highly enhanced, causing an increase in heat transfer. Another fact which needs attention is that for any given $\mathrm{Ca}$, there will be reduced oscillations of $\mathrm{Nu}_{\text {avg }}$, as $L / D$ increases. Alternately, for any given $L / D$, reducing Ca will eventually reduce oscillations in the value of $\mathrm{Nu}_{\text {avg }}$. In fact, when the cooler liquid travels a few times of its plug length, the peak and thus amplitude of $\mathrm{Nu}_{\text {avg }}$ oscillations are reduced.

Figure 15(i) shows the temporal variation of $\mathrm{Nu}_{\text {avg }}$ with $\mathrm{Ca}$ (for constant $L / D$ $=2$ ), and Fig. 15(ii) shows the temporal variation of $\mathrm{Nu}_{\text {avg }}$ with $L / D$ (for constant $\mathrm{Ca}=10^{-3}$ ), for water and glycerin plugs, respectively, under constant heat flux boundary conditions. The variation has the same trend as in the case of constanttemperature boundary conditions, except that the values of $\mathrm{Nu}_{\text {avg }}$ are increased by $35 \%$ under constant heat flux boundary conditions.

\section{CONCLUSIONS}

Transport parameters of isolated single liquid plugs moving inside dry capillary tubes is computationally analyzed. Flow near the interface is found to be threedimensional, and velocity profiles for all the components of velocity are reported. It is important to include the variations in the dynamic contact angles while determining the heat-transfer coefficient in isolated Taylor slug flows. Contact angle hysteresis plays a critical role in local transport behavior of liquid plugs 
(a) Water plug

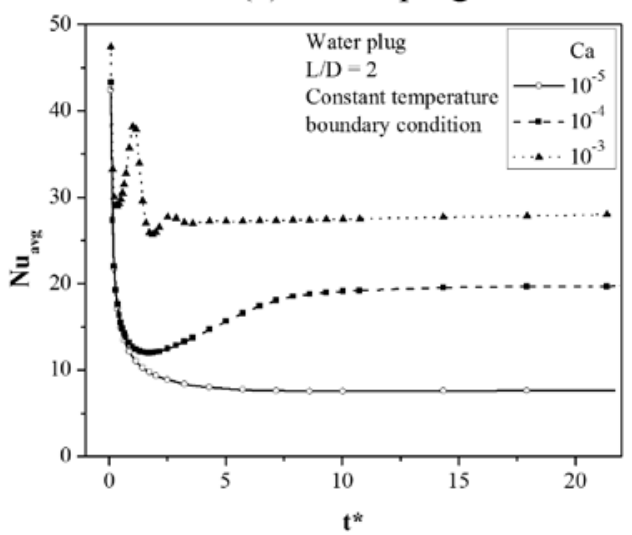

(b) Glycerin plug

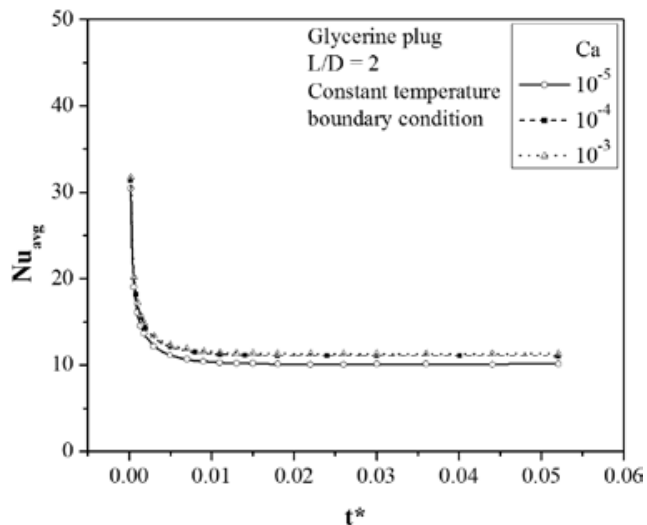

(i) Effect of $\mathrm{Ca}$, with constant $\mathrm{L} / \mathrm{D}=2$
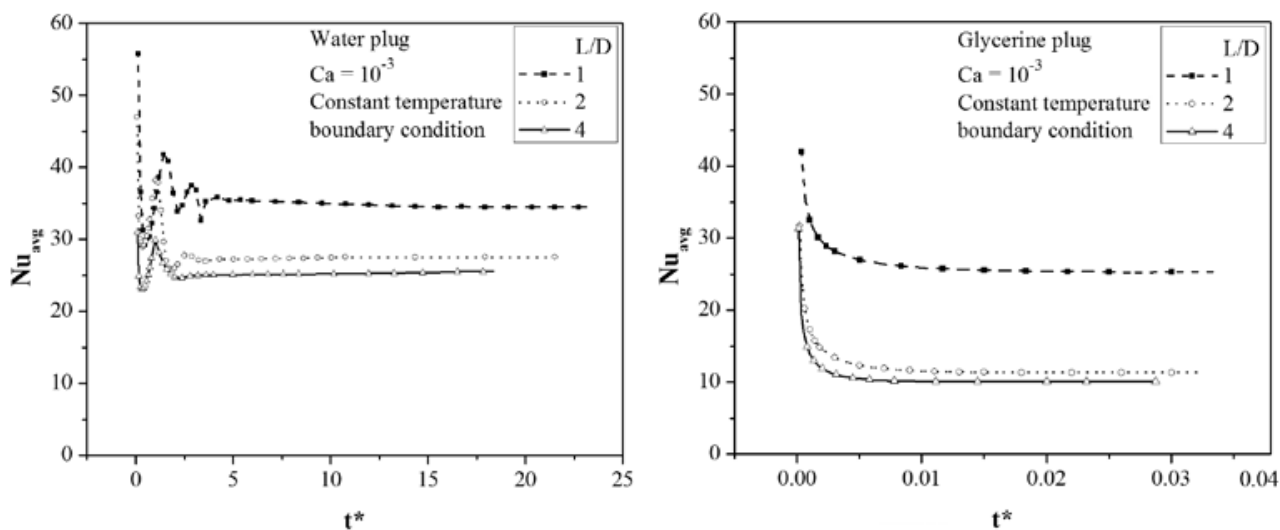

(ii) Effect of $\mathrm{L} / \mathrm{D}$, with constant $\mathrm{Ca}=10^{-3}$

FIG 14: Temporal variation of $\mathrm{Nu}_{\text {avg }}$ under constant wall temperature $T_{\mathrm{w}}=350 \mathrm{~K}$ for (a) a water plug and (b) glycerin plug. For these two cases: (i) variation with respect to flow $\mathrm{Ca}$ and constant $L / D=2$, and (ii) variation with respect to $L / D$ and constant $\mathrm{Ca}=10^{-3}$.

because of the resulting asymmetric vortices in the plug and increased internal circulations near the interface. Heat transfer in higher-Prandtl-number fluid is found to be diffusion dominant and internal circulations were not effected much compared to those in lower-Prandtl-number fluids such as water. Furthermore, the effect of $\mathrm{Ca}$ and $L / D$ ratio of the plug is investigated and it is observed that high velocity (high $\mathrm{Ca}$ ) and low $L / D$ ratio results in significantly higher heattransfer coefficients as compared to Poiseuille flow. Together, $\mathrm{Ca}, L / D, \operatorname{Pr}$, and hysteresis are the governing parameters of system transport. 
(a) Water plug

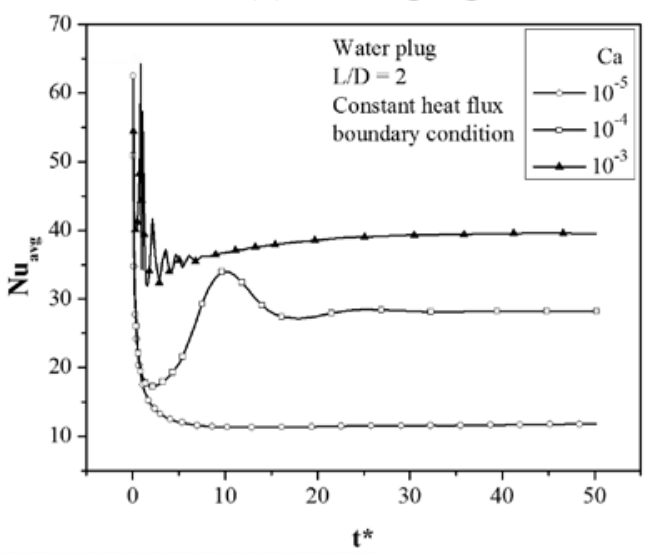

(b) Glycerin plug

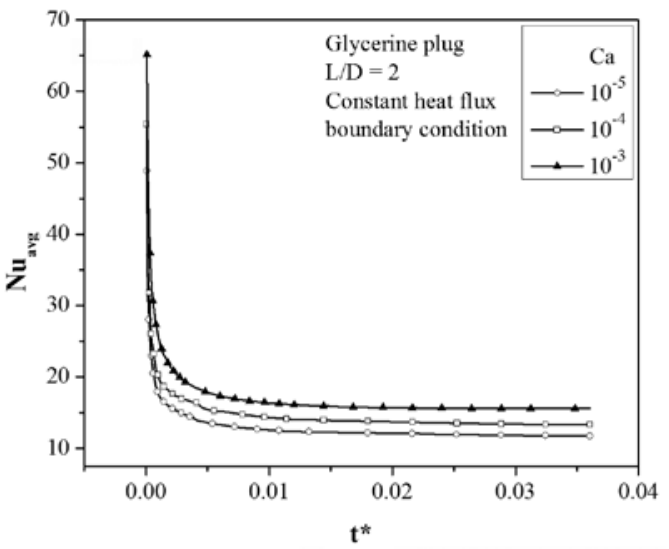

(i) Effect of Ca, with constant $\mathrm{L} / \mathrm{D}=2$
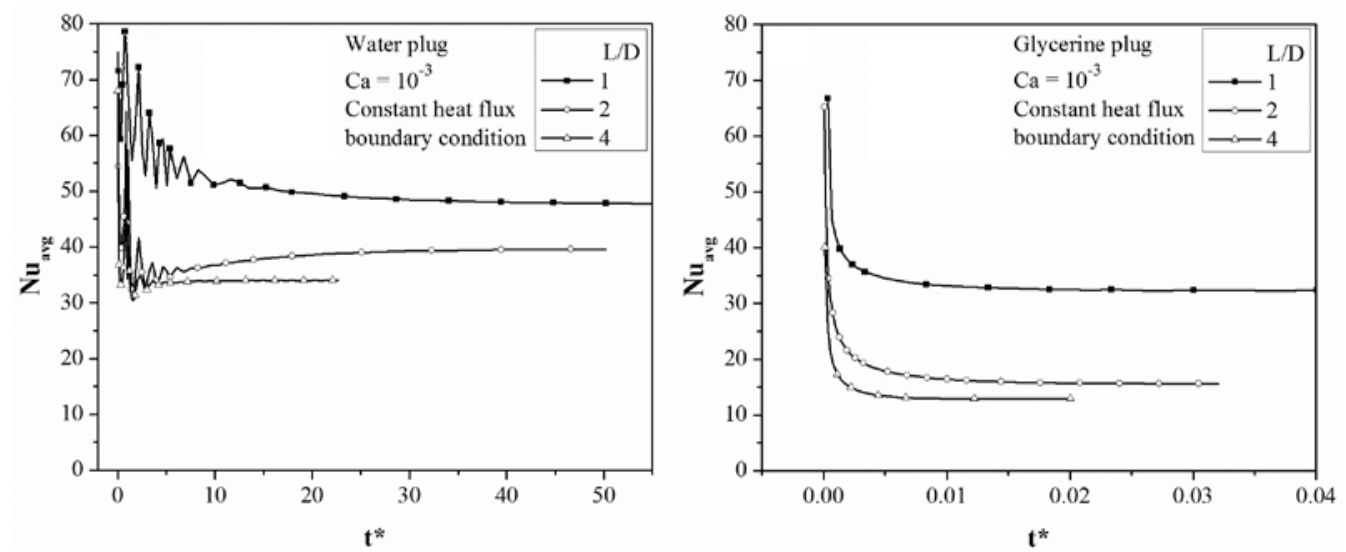

(ii) Effect of $\mathrm{L} / \mathrm{D}$, with constant $\mathrm{Ca}=10^{-3}$

FIG 15: Temporal variation of $\mathrm{Nu}_{\text {avg }}$ under constant heat flux at wall $q "=10,000 \mathrm{~W} / \mathrm{m}^{2}$ for (a) a water plug and (b) glycerin plug. For these two cases: (i) variation with respect to flow $\mathrm{Ca}$ and constant $L / D=2$, and (ii) variation with respect to $L / D$ and constant $\mathrm{Ca}=10^{-3}$.

\section{ACKNOWLEDGMENTS}

Financial support from the Indo-French Center for Promotion of Advanced Research (IFCPAR/CEFIPRA) is gratefully acknowledged (Project no. 4408-1). S.K. would also like to thank INSA Lyon, France, for extending an invitation as a visiting professor during May-July 2012. 


\section{REFERENCES}

Ansys-Fluent ${ }^{\circledR}$ Theory Guide Version-12.0, accessed Aug. 2012, http:// www1.ansys.com/customer/content/documentation/121/fluent/flth.pdf, 2009.

Barajas, A.M. and Panton, R.L., The effect of contact angle on two-phase flow in capillary tubes, Int. J. Multiphase Flow, vol. 19, no. 2, pp. 337-346, 1992.

Berthier, J., Microdrops and Digital Microfluidics, William Andrew, New York, USA, 2008.

Blake, T., The physics of moving wetting lines, J. Colloid Interface Sci., vol. 299, pp. 1-13, 2006.

Bretherton, F.P., The motion of long bubbles in tubes, J. Fluid Mech., vol. 10, pp. 166-188, 1961.

Chen, J.D., Measuring the film thickness surrounding a bubble inside a capillary, J. Colloid Interface Sci., vol. 109, pp. 341-349, 1986.

Cox, B.G., An experimental investigation of the streamlines in viscous fluid expelled from a tube, J. Fluid Mech., vol. 20, pp. 193-200, 1964.

Cubaud, T. and Ho, C.M., Transport of bubbles in square microchannels, Phys. Fluids, vol. 16, no. 12, pp. 4575-4585, 2004.

Fairbrother, F. and Stubbs, A.E., Studies in electro-endosmosis, VI. The 'bubble tube' method of measurement, J. Chem. Soc., vol. 1, pp. 527-529, 1935.

Fang, C., Hidrovo, C., Wang, F.M., Eaton, J., and Goodson, K., 3-D numerical simulation of contact angle hysteresis for microscale two-phase flow, Int. J. Multiphase Flow, vol. 34, no. 7, pp. 690-705, 2008.

Fermigier, M. and Jenffer, P., An experimental investigation of the dynamic contact angle in liquid-liquid systems, J. Colloid Interface Sci., vol. 146, no. 1, pp. 226-241, 1991.

Gunther, A., Khan, S.A., Thalmann, M., Trachsel, F., and Jensen, K.F., Transport and reaction in microscale segmented gas-liquid flow, Lab Chip, vol. 4, pp. 278-286, 2004.

Gupta, R., Fletcher, D.F., and Haynes, B.S., On the CFD modelling of Taylor flow in microchannels, Chem. Eng. Sci., vol. 64, pp. 2941-2950, 2009.

He, Q., Hasegawa, Y., and Kasagi, N., Heat transfer modelling of gas-liquid slug flow without phase change in a micro tube, Int. J. Heat Fluid Flow, vol. 31, pp. 126-136, 2010.

Hoffman, R., A study of advancing interface, J. Colloid Interface Sci., vol. 50, no. 2, pp. 228 $241,1974$.

Khandekar, S., Panigrahi, P.K., Lefevre, F., and Bonjour, J., Local hydrodynamics of flow in a pulsating heat pipe: A review, Front. Heat Pipes, vol. 1, p. 023003, 2010.

Kolb, W.B. and Cerro, R.L., Coating the inside of a capillary of square cross section, Chem. Eng. Sci., vol. 46, no. 9, pp. 2181-2195, 1991.

Lee, C.Y. and Lee, S.Y., Pressure drop of two-phase plug flow in round mini-channels: Influence of surface wettability, Exp. Therm. Fluid Sci., vol. 32, pp. 1716-1722, 2008.

Mehdizadeh, A., Sherif, S.A., and Lear, W.E., Numerical simulation of thermofluid characteristics of two-phase slug flow in microchannels, Int. J. Heat Mass Transfer, vol. 54, pp. 34573465, 2011.

Oliver, D.R. and Wright, S.J., Pressure drop and heat transfer in gas-liquid slug flow in horizontal tubes, Br. Chem. Eng., vol. 9, pp. 590-596, 1964. 
Rose, W. and Heins, R.W., Moving interfaces and contact angle rate-dependency, J. Colloid Sci., vol. 17, no. 1, pp. 39-48, 1962.

Rosengarten, G., Cooper-White, J., and Metcalfe, G., Experimental and analytical study of the effect of contact angle on liquid convective heat transfer in micro channels, Int. J. Heat Mass Transfer, vol. 49, pp. 4161-4170, 2006.

Schwartz, L.W., Princen, H.M., and Kiss, A.D., On the motion of bubbles in capillary tubes, $J$. Fluid Mech., vol. 172, pp. 259-275, 1986.

Serizawa, A., Feng, Z., and Kawara, Z., Two-phase flow in microchannels, Exp. Therm. Fluid Sci., vol. 26, pp. 703-714, 2002.

Shekhavat, S., Khandekar, S., and Panigrahi, P.K., Hydrodynamic study of an oscillating meniscus in a square mini-channel, Proc. in Micro/Nano-Scale Heat Mass Transfer Intl. Conf., Shanghai, China, 2009.

Tanner, L.H., The spreading of silicone oil drops on horizontal surfaces, J. Phys. D: Appl. Phys., vol. 12, pp. 1473-1484, 1979.

Taylor, G.I., Deposition of a viscous fluid on the wall of a tube, J. Fluid Mech., vol. 10, pp. 161-165, 1961.

Thulasidas, T.C., Abraham, M.A., and Cerro, R.L., Flow patterns in liquid slugs during bubbletrain flow inside capillaries, Chem. Eng. Sci., vol. 52, no. 17, pp. 2947-2962, 1997.

Tripathi, A., Khandekar, S., and Panigrahi, P.K., Oscillatory contact line motion inside capillaries, Proc. of the 15th Intl. Heat Pipe Conf., Clemson, SC, 2010.

Triplett, K.A., Ghiaasiaan, S.M., Abdel-Khalik, S.I., and Sadowski, D.L., Gas-liquid two-phase flow in micro channels, Int. J. Multiphase Flow, vol. 25, no. 3, pp. 377-394, 1999.

Walsh, P.A., Walsh, E.J., and Muzychka, Y.S., Prandtl and capillary effects on heat transfer performance within laminar liquid-gas slug flows, Int. J. Heat Mass Transfer, vol. 54, pp. 4752-4761, 2011.

Zhao, T.S. and Bi, Q.C., Co-current air-water two phase flow patterns in vertical triangular micro channels, Int. J. Multiphase Flow, vol. 27, no. 5, pp.765-782, 2001.

Zheng, Y., Fujioka, H., and Grotberg, J.B., Effect of gravity, inertia and surfactant on steady plug propagation in a two-dimensional channel, Phys. Fluids, vol. 19, p. $082107(1-16), 2007$. 